\title{
Eulerian-Lagrangian model for gas-evolving processes based on supersaturation
}

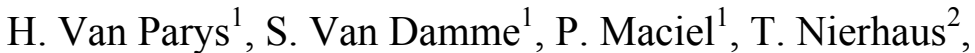 \\ F. Tomasoni ${ }^{3}$, A. Hubin ${ }^{1}$, H. Deconinck ${ }^{2} \&$ J. Deconinck ${ }^{1}$ \\ ${ }^{1}$ Vrije Universiteit Brussel, \\ Research Group of Electrochemical and Surface Engineering, Belgium \\ ${ }^{2}$ von Karman Institute for Fluid Dynamic, \\ Department of Aeronautics and Aerospace, Belgium \\ ${ }^{3}$ von Karman Institute for Fluid Dynamic, \\ Department of Environmental and Applied Fluid Dynamics, Belgium
}

\begin{abstract}
Predicting the macro-scale behaviour of gas bubbles and understanding the coupling between bubbles and fluid flow is essential if one wants to describe the local concentration of all electrolyte components and the current density distribution at an electrode surface. The presented two-phase model uses an Eulerian-Lagrangian approach to simulate the hydrodynamic phenomena occurring in the bubble-laden flow. The model describes the continuous electrolyte phase via the incompressible Navier-Stokes equations, while the trajectories of the gas bubbles are tracked sequentially in space and time. The electrochemical behaviour of the reactor is described using the Multi-Ion Transport and Reaction Model (MITReM), which considers for all relevant species the effect of convection, diffusion and migration and homogeneous reactions. For the electrode reactions, Butler-Volmer kinetics are used. The MITReM provides the concentration of dissolved gas at the electrode and in the electrolyte. At predefined nuclei gas bubbles are formed proportional to the local supersaturation. After a certain time the spherical bubbles are large enough to become detached bubbles. While the bubbles are growing on the electrode surface, they block the surface and therefore also influence the current density distribution. The first steps in view of the validation of the proposed two-phase model against experiments are reported.
\end{abstract}

Keywords: gas evolving electrode, supersaturation, bubble nucleation and growth, hydrogen evolution. 


\section{Introduction}

Many electrochemical applications, such as the electroplating of chrome, zinc or nickel, suffer from unwanted side-effects related to gas production on electrodes. In other processes gas formation is the only aim.

In all cases the formation of gas bubbles has an effect on the mass transport of the electrochemically active species in the process [1]. The gas bubbles sticking at the nucleation sites block the electrode and decrease the active surface [2-6], while the bubbles that are detached into the electrolyte solution change the conductivity of the electrolyte [7-9]. Furthermore, momentum exchange occurs between the bubbles and the surrounding electrolyte, affecting the motion of the electrolyte [10].

The mechanism of bubble formation by nucleation requires supersaturation of the dissolved gas [11-13] and a nucleus radius greater than the critical [7]. The main sources of heterogeneous nucleation are usually surface irregularities capable of containing entrapped gas, e.g. pits and scratches. The bubbles typically develop over the electrode surface, grow in size until they reach a break-off diameter and subsequently detach into the electrolyte. After detachment, some residual gas remains at the nucleation site and another bubble will form at the same place $[2,13,14]$. In most two-phase flow simulations [15-19], it is assumed that bubbles detach with a constant diameter, although from experiments $[20,21]$ it is know that electrochemically formed bubbles show a size distribution.

Present models are based on primary and secondary current density distributions, with the gas production based on an empirical correlation with current density [15-19]. These models don't solve for concentrations. Therefore they cannot describe properly the bubble formation mechanism.

We propose a new macroscopic model based on supersaturation, providing a physically relevant link between the dissolved gas production and the bubble formation mechanism.

\section{Eulerian fluid flow and ion transport model}

For the simulation of gas-evolving electrochemical processes, a new approach is proposed that combines numerical models for electrolyte flow, ion transport and gas evolution. The mass and momentum conservation of the electrolyte flow is modelled by the incompressible Navier-Stokes equations, solving for the fluid velocity $\mathbf{u}$ and the pressure $p$ :

$$
\begin{gathered}
\nabla \cdot \mathbf{u}=0, \\
\frac{\partial \mathbf{u}}{\partial t}+(\mathbf{u} \cdot \nabla) \mathbf{u}+\frac{1}{\rho} \nabla p-v \nabla^{2} \mathbf{u}=0 .
\end{gathered}
$$

In equation (2), $\rho$ is the electrolyte density and $v$ is the viscosity.

Based on the Multi-Ion Transport and Reaction Model (MITReM) [22,23], we can state a balance equation for each species in the system: 


$$
\frac{\partial c_{i}}{\partial t}=-\nabla \cdot \mathbf{N}_{\mathbf{i}}+\sum_{r=1}^{R} s_{i r} v_{r}
$$

with $c_{i}$ the concentration of species $i$. The source term on the right hand side of equation (3) comes from the homogeneous reactions, where $v_{r}$ is the rate of reaction $r$ and $s_{i r}$ is the stoichiometric coefficient of species $i$ in that reaction. The flux $\mathbf{N}_{\mathbf{i}}$ is given by convection, diffusion and migration as follows:

$$
\mathbf{N}_{\mathbf{i}}=-c_{i} \mathbf{u}+D_{i} \nabla c_{i}+\frac{z_{i} F D_{i}}{R T} c_{i} \nabla U
$$

with $D_{i}$ the diffusion coefficient, $z_{i}$ the charge, $F$ Faraday's constant, $R$ the universal gas constant, $T$ the temperature and $U$ the potential. The flux perpendicular to an electrode is zero or given by the heterogeneous reactions

$$
\mathbf{N}_{\mathbf{i}} \cdot \mathbf{1}_{\mathbf{n}}=\sum_{e=1}^{E} s_{i e} v_{e} .
$$

For these reactions, the Butler-Volmer kinetics are used [23].

\section{Supersaturation model}

In this model, bubbles are formed by consuming dissolved gas, with supersaturation as the driving mechanism for bubble nucleation and growth. Supersaturation is given by $c-c_{\text {sat }}$, where $c_{\text {sat }}$ is the saturation concentration of a species [10]. In a first approach, the bubble growth rate is considered as a single heterogeneous reaction taking place continuously with a linear rate law:

$$
v=k\left(c-c_{\text {sat }}\right) \text { if } c-c_{\text {sat }}>0
$$

with $k$ the rate constant. As it is known that gas bubbles are formed at nuclei, the produced gas volume needs to be attributed to bubbles at discrete nucleation sites. Here it is assumed that the nuclei are located at predefined places. Additional fundamental research is needed to justify or correct this rather simple assumption. In practice many parameters are influencing the number of nuclei e.g. surface roughness, surface tension, contact angle, current density, etc. A predefined bubble diameter at which detachment will occur is assigned to every nucleus. Actually, this is done randomly in such a way that the measured lognormal bubble diameter distribution is reproduced. As in practice, also the bubble diameter depends on flow velocity, current density, roughness, etc., this bubble size distribution is measured under the same working conditions as the simulation. It is clear that this approach can be improved by incorporation of more details. Finally, to every nucleus a given surface is assigned from where the gas is feeding the growing bubble. In this way the local gas production rate can be integrated over time and space. So, the amount of produced gas is accumulated in a growing spherical bubble having a zero contact angle with the electrode. The projection of the actual bubble size on the electrode surface is assumed to cover the surface such that the local active electrode surface is reduced resulting in a changed local current density. All this is calculated in an Eulerian way. Once the bubble diameter has reached its assigned diameter, the bubble is released and transferred to the Lagrangian bubble tracker and a new 
bubble diameter is randomly assigned to that nucleus. Also here much better and more refined models can be conceived.

\section{Lagrangian bubble tracking model}

Every detached bubble enters the electrolyte and a Lagrangian tracking procedure is used to update the velocities and positions of all dispersed gas bubbles in the electrolyte at each time step of the Navier-Stokes solver. From Newton's second law, an equation of motion can be obtained for every bubble, based on the formulation stated in [24]. Together with the relation between the particle's position and velocity, a set of two ordinary differential equations in three space dimensions can be formed in order to update the bubble trajectory

$$
\begin{aligned}
& \frac{\partial \mathbf{x}}{\partial t}=\mathbf{v} \\
& \frac{\partial \mathbf{v}}{\partial t}=\frac{1}{\tau_{b}}(\mathbf{u}-\mathbf{v})+3 \frac{D \mathbf{u}}{D t}+2 C_{L}((\mathbf{u}-\mathbf{v}) \times \omega)-2 \mathbf{g},
\end{aligned}
$$

where $\mathbf{v}$ is the bubble velocity, $\mathbf{u}$ is the fluid velocity and $\boldsymbol{\omega}$ the fluid vorticity at the bubble position. The derivative $\partial / \partial t$ follows the moving bubbles in time, while $D / D t$ is the total acceleration of the carrier fluid as seen by the bubble, evaluated at the bubble position $\mathbf{x}$. The terms of the right hand side of equation (8) represent the forces acting on the bubble. From left to right, these are the drag force, the added mass force, the Saffman lift force $\left(C_{L}\right.$ is the lift coefficient) and the force due to gravity $\mathbf{g}$, i.e. the buoyancy force. The drag force represents an inter-phase momentum transfer and reduces the velocity difference between the phases, depending on the bubble response time $\tau_{b}$ for Stokes flow:

$$
\tau_{b}=\frac{d^{2}}{36 v},
$$

where $d$ is the bubble diameter. If the bubble accelerates relative to the carrier fluid, it will immediately experience a deceleration due to its inertia to the other phase. This effect is known as the added mass force and represents the amount of volume of the fluid displaced by the bubble in its relative motion. The Saffman lift force represents the lift on the bubble induced by a shear velocity, where $C_{L}$ is the lift coefficient. In case of a small spherical bubble, $C_{L}$ takes a value of 0.53 , as proposed in [25].

The bubbles occurring in the present electrochemical system are small enough to fulfil the criterion of Stokes flow. Thus, turbulent wakes arising behind bubbles can be neglected [26]. Furthermore, we consider all bubbles in the present simulations as small, non-deformable and rigid spheres. This hypothesis holds for bubbles of low Eötvös numbers Eo:

$$
E o=\frac{\Delta \rho d^{2} g}{\sigma} \text {. }
$$

Here, $\sigma$ denotes the surface tension of the bubble and $\Delta \rho$ the difference in density of the two phases. The sphericity assumption of bubbles is valid when 
both $E o$ and the bubble Reynolds number $R e_{d}=\mathbf{v} d / v$ are of the order of 10 or lower. For hydrogen bubbles of sizes below $300 \mu \mathrm{m}$, this restriction is valid and thus sphericity is assured [27].

Equations (7) and (8) form a system of six ordinary differential equations in three space dimensions for each individual bubble. This system is integrated in time using a Crank-Nicholson scheme, which provides second order accuracy. Sequential tracking of all bubbles in the system is performed at each time step of the Navier-Stokes solver.

\section{The electrochemical system}

For the electrolyte solution a $1 \mathrm{M} \mathrm{Na}_{2} \mathrm{SO}_{4}$ solution is taken. The $\mathrm{pH}$ is adjusted to $\mathrm{pH} 2.5$ by adding concentrated $\mathrm{H}_{2} \mathrm{SO}_{4}$. The diffusivities and bulk concentrations of all species are given in table 1 .

Dissolved hydrogen formation occurs on the cathode and dissolved oxygen on the anode according to reactions (7) and (8) respectively:

$$
\begin{gathered}
2 \mathrm{H}^{+}+2 e^{-} \rightarrow \mathrm{H}_{2}^{(l)} \\
2 \mathrm{H}_{2} \mathrm{O} \rightarrow \mathrm{O}_{2}^{(l)}+4 \mathrm{H}^{+}+4 e^{-}
\end{gathered}
$$

The electrode reactions kinetic parameters are indicated in table 2.

Table 1: $\quad$ Electrolyte species diffusivity and bulk concentrations [23,28,29].

\begin{tabular}{ccc}
\hline Species & $\mathbf{D}\left[\mathbf{1 0}^{-9} \mathbf{m}^{\mathbf{2}} \mathbf{s}^{-\mathbf{1}}\right]$ & $\mathbf{C}_{\text {bulk }}\left[\mathbf{m o l ~ \mathbf { m } ^ { - 3 } ]}\right.$ \\
\hline $\mathrm{Na}^{+}$ & 1.334 & 2000. \\
$\mathrm{SO}_{4}{ }^{2-}$ & 1.065 & 1000. \\
$\mathrm{HSO}_{4}{ }^{-}$ & 1.330 & 3.16 \\
$\mathrm{H}^{+}$ & 9.312 & 3.16 \\
$\mathrm{OH}^{-}$ & 5.260 & $3.1610^{-9}$ \\
$\mathrm{H}_{2} \mathrm{O}$ & 2.300 & 55000. \\
$\mathrm{H}_{2}{ }^{(\mathrm{l})}$ & 4.870 & 0. \\
\hline
\end{tabular}

Table 2: $\quad$ Electrode reactions kinetic parameters deduced from polarization curves on a rotating disc electrode.

\begin{tabular}{rrrrr}
\hline $\mathbf{E 0}[\mathbf{V}]$ & $\mathbf{k}_{\text {oxi }}\left[\mathbf{m ~ s}^{-1}\right]$ & $\mathbf{k}_{\text {red }}\left[\mathbf{m ~ s}^{-1}\right]$ & $\boldsymbol{\alpha}_{\mathbf{o x i}}[\mathbf{1}]$ & $\boldsymbol{\alpha}_{\text {red }}[\mathbf{1}]$ \\
\hline $2 H^{+}+2 e^{-} \rightarrow H_{2}^{(l)}$ & & & & \\
0.000 & - & $5.70010^{-13}$ & - & 0.990 \\
\hline $2 H_{2} O \rightarrow O_{2}^{(l)}+4 H^{+}+4 e^{-}$ & & & \\
1.230 & $5.31210^{-11}$ & - & 0.045 & - \\
\hline
\end{tabular}


For simplicity and without any loss of generality oxygen gas evolution is not considered and therefore the dissolved oxygen was excluded from the model. The transfer from dissolved hydrogen to gaseous hydrogen is as follows

$$
H_{2}^{(l)} \rightarrow H_{2}^{(g)}
$$

with kinetics according to eq. (6), a rate constant $\mathrm{k}=10^{-6} \mathrm{~m} \mathrm{~s}^{-1}$ and the solubility of $\mathrm{H}_{2}$ in water $c_{\text {sat }}=0.00075 \mathrm{~mol} . \mathrm{m}^{-3}$. The hydrogen gas evolution reaction is considered along the whole lower boundary (including insulators).

\section{Results}

It has been shown [30] that the kinetic constant $k$ of reaction (6) plays an important role. For high reaction rates, the local gas evolution is almost proportional to the local current density, whereas for lower reaction rates one can have a substantial difference between the local bubble formation rate and the local current density. Indeed much dissolved gas enters the solution by diffusion and convection. It is worth mentioning that in this situation gas evolution can also occur on nuclei that do not belong to the cathode.

In view of evaluation of the simulation approach, a parallel flow reactor was build with the following dimensions: length $1 \mathrm{~m}$, width $10 \mathrm{~cm}$ and height $1 \mathrm{~cm}$. The inlet is made large enough to assure steady flow along all electrodes. The electrodes are $10 \mathrm{~cm}$ wide, $5 \mathrm{~cm}$ long and configured such that the cathode is fully optically accessible (figure 1). This involves that they are not aligned and that the current density distribution is intentionally non-uniform.
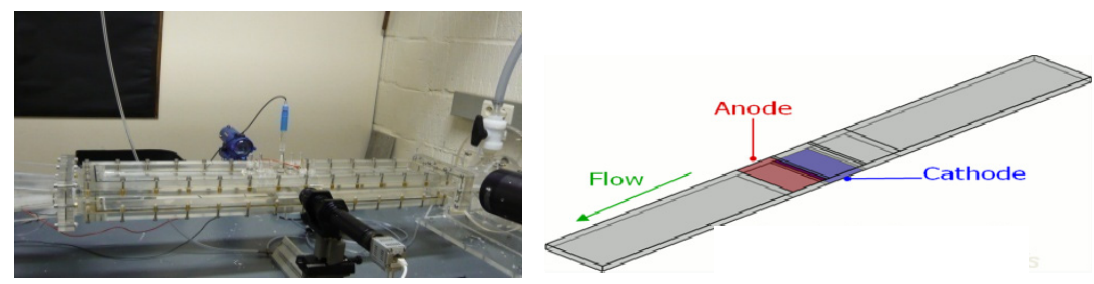

Figure 1: The reactor with unaligned electrode configuration.

As the upstream anode edge is situated $1.5 \mathrm{~cm}$ from the cathode's downstream edge, bubbles formed at the anode are neglected. A typical current density distribution along the cathode is given in figure 2 . The small peak on the right hand side of the curve is attributed to the proton reduction to $\mathrm{H}_{2}$ (see eq. (11)). However, the $\mathrm{H}^{+}$-ions become rapidly depleted and as a consequence, the $\mathrm{pH}$ rises in the immediate vicinity of the electrode surface making the reduction from $\mathrm{H}_{2} \mathrm{O}$ molecules thermodynamically more favourable (see eq. (12)).

The bubble size distribution was also measured at a cell potential of $3.3 \mathrm{~V}$ and is given in figure 3 . To that purpose backlighting or shadowgraphy, being an in situ and non-intrusive optical measurement technique, was used.

In backlighting, the bubble is illuminated by a diffuse light source from one side and its shadow is imaged with a high speed camera. After calibration the 


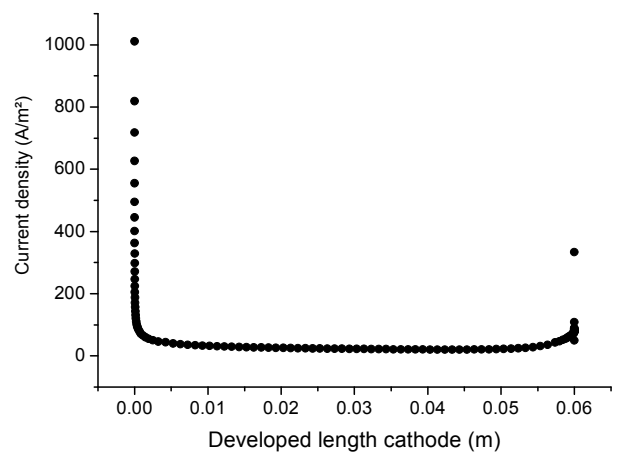

Figure 2: Current density distribution along the cathode for an applied total voltage of $3.3 \mathrm{~V}$.

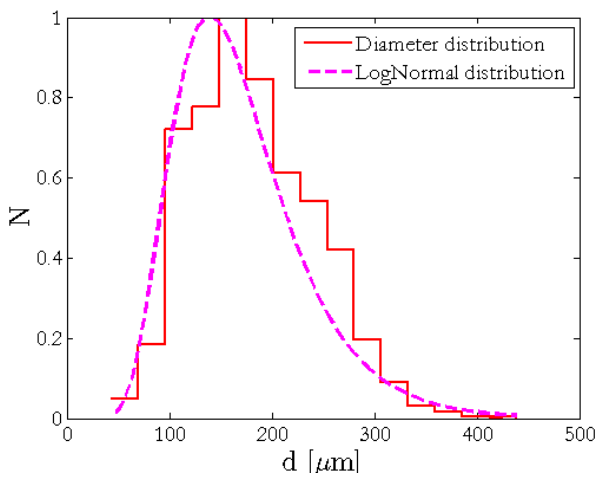

Figure 3: Hydrogen bubble size distribution for an applied total voltage of $3.3 \mathrm{~V}$ and a mean flow of $0.2 \mathrm{~m} / \mathrm{s}$.

projected shadow image is a measure for the bubble diameter. A lognormal distribution is fitted to the bubble diameter distribution. A mean diameter of $170 \mu \mathrm{m}$ with a standard deviation of $64 \mu \mathrm{m}$ is obtained. As explained above, this bubble distribution is used as input data for the simulations.

In order to avoid calculating the whole transient process, steady state flow and MITReM conditions are calculated first. From this situation on the simulation of time dependent bubble evolution is started. A two-way interaction between bubbles and flow is considered. This means that the combined effect of the influence of the fluid flow on the bubble trajectories and the effect of the bubble movement on the fluid flow is taken into account. In figure 4 simulated situations at several time steps are shown. The mean flow is $0.2 \mathrm{~m} / \mathrm{s}$. The cathode is on the right. 


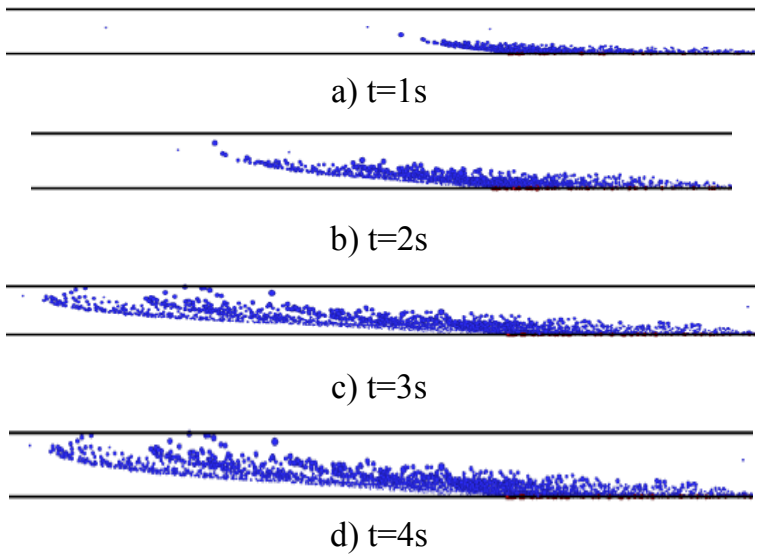

Figure 4: $\quad$ Side view of simulated hydrogen bubble dispersion for different time steps.

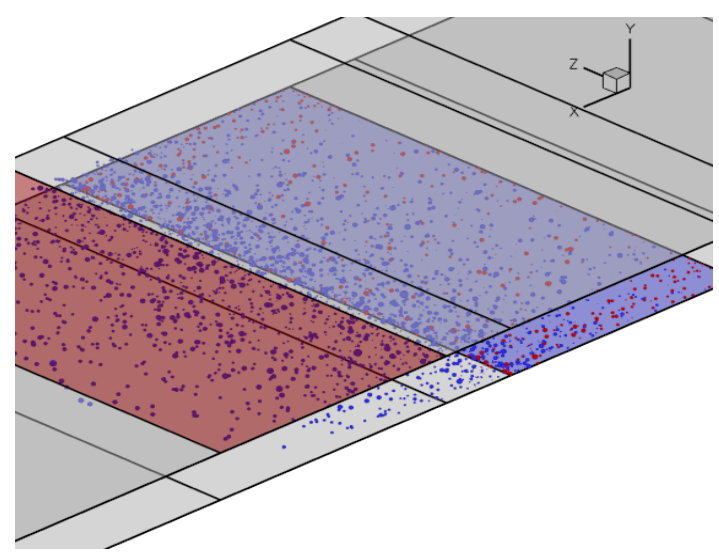

Figure 5: Isometric view of simulated hydrogen bubble dispersion for $\mathrm{t}=2 \mathrm{~s}$. The mean flow is $0.2 \mathrm{~m} / \mathrm{s}$.

In figure 5 an isometric view is given for $\mathrm{t}=2 \mathrm{~s}$. In this figure bubbles on the surface are coloured in dark grey whereas bubbles in the flow are marked in medium grey. It is clearly observed that more bubbles are formed at the cathode edge where the current density is larger and that larger bubbles are rising faster. Unfortunately these simulated bubble dispersions cannot be directly compared with measurements as in reality always a transient current density phenomenon takes place when the voltage is applied.

Also steady state calculations, performed in different applied flow and potential conditions, need still to be compared in full detail with measurements performed in the same conditions. It is believed however that it is already proven that the concepts work and will open new possibilities for bubble simulations in electrochemical reactors. 


\section{Conclusions}

Models that directly relate the gas bubble flux to the current density implicitly assume that the nucleation and growth of bubbles is very fast. This assumption is not needed in the present modelling approach as gas evolution is related to supersaturation instead of current density.

Although so far still rather drastic simplifications have been introduced, it is clear that the presented method offers new ways to model electrochemical gas evolution, at least at moderate gas fractions where bubbles are dispersed in the solution.

Future work will be directed towards a more detailed description of nuclei and bubble formation, bubble size determination, all influenced by local quantities such as surface roughness, charge distribution, surface tension (depending on local concentrations), concentration of dissolved gas.

\section{Acknowledgement}

The authors thank the Flemish Institute for support of Scientific-Technological Research in Industry (IWT SBO contract number 040092, project acronym: MuTEch and IWT post-doc research mandate).

\section{References}

[1] J.E. Funk, J. Electrochem. Soc. 116 (1969) 48.

[2] H. Vogt, Electrochim. Acta 25 (1980) 527.

[3] P.J. Sides, C.W. Tobias, J. Electrochem. Soc. 127 (1980) 288.

[4] M. Krenz, L. Müller, A. Pomp, Electrochim. Acta 31 (1986) 723.

[5] J. Dukovic, C.W. Tobias, J. Electrochem. Soc. 134 (1987) 331.

[6] H. Vogt, R.J. Balzer, Electrochim. Acta 50 (2005) 2073.

[7] H. Vogt in: J.O'M. Bockris (Ed.), B. E. Conway (Ed.), E. Yeager (Ed.), Comprehensive Treatise of Electrochemistry, vol. 6, Plenum Press, London, 1983, Ch.7.

[8] P. Byrne, P. Bosander, O. Parhammar, E. Fontes, J. Appl. Electrochem. 30 (2000) 1361.

[9] G. Kreysa, M. Kuhn, J. Appl. Electrochem. 15 (1985) 517.

[10] E. Delnoij, J.A.M. Kuipers, W.P.M. Van Swaaij, Chem. Eng. Sci. 54 (1999) 2217.

[11] D. Kashchiev, A. Firoozabadi, J. Chem. Phys. 98 (1993).

[12] J. Eigeldinger, H. Vogt, Electrochim. Acta 45 (2000) 4449.

[13] S. Lubetkin, Langmuir (2003) 2575.

[14] S. Lubetkin, Electrochim. Acta 48 (2002) 357.

[15] P. Mandin, J. Hamburger, S. Bessou, G. Picard, Electrochim. Acta 51 (2005) 1140.

[16] R. Wüthrich, C. Comninellis, H. Bleuler, Electrochim. Acta 50 (2005) 5242.

[17] M.D. Mat, K. Aldas, Int. J. Hydrogen Energy 30 (2005) 411. 
[18] K. Aldas, Applied Mathematics and Computation 154 (2004) 507.

[19] G. H. Kelsall, G. Li in: R. Woods (Ed.), F.M. Doyle (Ed.), Int. Symp. on Electrochemistry in Mineral and Metal Processing V, Electrochem. Soc., N.J., (2000) 303.

[20] S. Dehaeck, Ph.D. Thesis, Ghent University, Belgium

[21] P. Boissoneau and P. Byrne, J. Appl. Electrochem. 30 (2000) 767

[22] L. Bortels, J. Deconinck, B. van den Bossche, J. Electroanal. Chem. 404 (1996) 15.

[23] J. Newman, Electrochemical Systems, 3rd ed., Prentice-Hall, Englewood Cliffs, NJ, 2004.

[24] M.R. Maxey and J.J. Riley, Phys. Fluids 26 (1983) 883

[25] T.R. Auton, Ph.D. Thesis, University of Cambridge, United Kingdom (1983)

[26] A.W.G. de Vries, A. Biesheuvel and L. van Wijngaarden, Int. J. Multiphase Flow 28 (2002) 1823

[27] R. Clift, J.R. Grace and M.E. Weber, in Bubbles, drops and particles, 2nd Ed, Academic Press, New York, United States (2005)

[28] B.E. Conway, Electrochemical Data, Elsevier, Amsterdam, 1952.

[29] V. P. Arkhipov, M. I. Emel'yanov, F. M. Samigullin, N. K. Gaisin, J. Struct. Chem. 19 (1979) 709.

[30] P. Maciel, T. Nierhaus, S. Van Damme, H. Van Parys, J. Deconinck, A. Hubin, Electrochemistry Communications 11 (2009) 875 